Polymer Journal, Vol. 4, No. 6, pp 651-656 (1973)

\title{
Polymerization of Acetaldazine
}

\author{
Mikiharu Kamachi and Shunsuke Murahashi \\ Department of Polymer Science, Faculty of Science, \\ Osaka University, Toyonaka, Osaka, Japan.
}

(Received November 17, 1972)

\begin{abstract}
Addition polymerization of the carbon-nitrogen double bond in acetaldazine, the azomethine analog of 2,4-hexadiene, has been studied. No polymer was obtained by radical initiator, while an oligomer with molecular weight of 800-1200 was obtained by $n$-butyllithium in aprotic solvents. The structure of the oligomer thus obtained was investigated by IR, UV and mass spectroscopies and chemical functional tests. The oligomer was composed of 1,2-addition structure $(-\mathrm{CH}-\mathrm{N}-)$ and 1,4-addi-

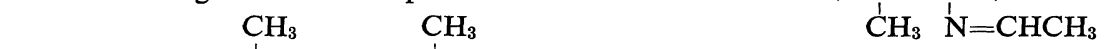
tion structure $(-\stackrel{\dot{C}}{\mathrm{H}}-\mathrm{N}=\mathrm{N}-\mathrm{C} H-)$. It was confirmed that the comparison of the number of oligomers with the number of charged initiator indicated an easy occurrence of a proton transfer from the acetaldazine monomer to the propagating anion. In addition, the anionic polymerizability of acetaldazine is discussed using various initiators for anionic polymerization in comparison of the half-wave potential of acetaldazine with that of various vinyl compounds and butadiene.

KEY WORDS Acetaldazine / Anionic Polymerization / Radical Polymerization / Micro Structure / Half-Wave Potential / 1,2-Addition / 1,4-Addition / Oligomer /
\end{abstract}

Only a few addition polymerizations of the compounds having carbon - nitrogen double bonds have been reported. ${ }^{1-6}$ In 1959, Shashoua showed that isocyanates were polymerized to 1-nylons of high molecular weight by a variety of anionic catalysts at low temperatures. This was the first unequivocal demonstration of the polymerization of the carbon-nitrogen double bond. Since then, information concerning the anionic polymerization of isocyanates has been reported..$^{2-5}$ In addition, the anionic polymerization of carbodiimide was reported by Robinson ${ }^{6}$ in 1964; the carbon-nitrogen double bonds of these compounds have greater $\pi$-delocalization than the isolated carbon-nitrogen double bond. If this $\pi$-delocalization is an important factor for the polymerization of carbon-nitrogen double bonds, the polymerization of azine compounds could be expected to occur. In this regard, Neureiter ${ }^{7}$ has reported that formaldazine spontaneously polymerized without a catalyst at room temperature, and the type of polymerization which took place was not specified. On the other hand, papers concerning the reaction of azine compounds with organometallics have been presented, ${ }^{8,9}$ while the occurrence of a polymer in these reactions was not mentioned.

In this paper, the polymerization of acetaldazine by various catalysts and the investigation of the structure of the oligomers obtained are described, where both 1,2-addition and 1,4-addition take place. In addition, the anionic polymerizability of acetaldazine is discussed.

\section{EXPERIMENTAL}

\section{Monomer}

Acetaldazine was prepared from hydrazine hydrate and acetaldehyde according to the procedure of Curtius, ${ }^{10}$ and purified by reduced pressure distillation through a Widmer Column under nitrogen.

\section{Solvents}

Benzene, toluene, dioxane and heptane were purified in the usual manner ${ }^{11}$ and were dried over metallic sodium. Tetrahydrofuran and dimethoxyethane were refluxed over metallic sodium and distilled into an ampoule on a high vacuum system. 


\section{Catalysts}

According to the method of Ziegler, ${ }^{12} n$-butyllithium (BuLi) was prepared in $n$-heptane, and the concentration $(6.6$ mol\%) was determined by double titration. ${ }^{13} n$-Butylmagnesium chloride was prepared by the reaction of magnesium with $n$-butyl chloride and was titrated by the method of Gilman. ${ }^{14}$ Monosodium benzophenone was prepared by adding a tetrahydrofuran solution of benzophenone to an ampoule coated with a fresh sodium film through a breakable seal under high vacuum. The concentration was checked by the back titration method. ${ }^{15}$ Sodium naphthalene was formed by distilling tetrahydrofuran from a graduated glass into an evacuated flask which contained a known amount of naphthalene and was coated with an excess of sodium film. ${ }^{16}$ This filtrate was titrated by the method of Gilman. ${ }^{14}$

$2,2^{\prime}$-Azobisisobutyronitrile was purified by recrystallization from $95-\%$ ethanol. Benzoyl peroxide was purified by fractional precipitation from a chloroform solution, using ethyl alcohol as precipitant.

\section{Polymerizations}

A polymerization ampoule with a nitrogen inlet and stopcock was connected to a highvacuum system, evacuated, and then flushed with nitrogen from the nitrogen inlet. The ampoule was removed and then a solution of the catalyst was injected by a hypodermic syringe into the ampoule under nitrogen. The ampoule was again connected to a high-vacuum system and then weighed amounts of solvent and acetaldazine, which were previously dried and degassed, were distilled into it. After the ampoule was sealed off, it was placed in a thermostat. After a given time, the contents were put into ether containing a few percent of methanol. The ether solution was shaken with distilled water and dried by anhydrous potassium carbonate. A polymer was obtained from the ether solution by evaporation. In the polymerizations by sodium naphthalene or sodium benzophenone, residual naphthalene and benzophenone were separated by the extraction with petroleum benzin.

The elementary analysis of the water-insoluble oligomer (mol wt, 730) obtained from butyllithium at $-78^{\circ} \mathrm{C}$ was as follows

Calcd for $\mathrm{C}_{36} \mathrm{H}_{74} \mathrm{~N}_{16}$ :

$$
\text { C, 59.14; H, 10.20; N, } 30.66 \text {. }
$$

Found: C, 58.67; H, 9.82; N, 30.48.
The elementary analysis of the oligomer (mol wt, 730) was calculated by assuming a terminal butyl group originated from the initiator.

In the radical polymerization, a polymerization ampoule, in which 2,2' -azobisisobutyronitrile or benzoyl peroxide was previously placed, was connected to a high-vacuum system; acetaldazine and the solvent were distilled into it. After the ampoule was sealed, it was placed in a thermostat. No polymer or oligomer was obtained. The monomer was recovered in $93 \%$ by vacuum distillation.

Chemical Functional Tests for the Determination of the Structure of the Oligomer

After the treatment of $0.5 \mathrm{~g}$ of oligomer with $20 \mathrm{ml}$ of $0.1 \mathrm{~N} \mathrm{HCl}$, the volatile product was added to an excess of 2,4-dinitrophenylhydrazine reagent $^{17}$ and Tollen's reagent ${ }^{18}$ by high-vacuum distillation. The residual oligomer was isolated by evaporating the solvent and the volatile product completely. The oligomer was neutralized by $0.1 \mathrm{~N} \mathrm{NaOH}$ and was put into an excess of Tollen's reagent. ${ }^{18}$

Pyrolysis of the Oligomer

A $0.3 \mathrm{~g}$ sample of oligomer was put into a $10 \mathrm{~m} l$ ampoule equipped with a sample tube for the mass spectrometer, evacuated under highvacuum system, and heated at $150^{\circ} \mathrm{C}$ for $2 \mathrm{hr}$. Gas obtained from its pyrolysis was characterized by mass spectrometry.

\section{Measurements}

The IR spectra were measured on a Nippon Bunko IRS infrared spectrometer. The electronic spectra were measured on a Hitachi model EPS-2 spectrometer. The mass spectrum was measured on a Hitachi model HRU-10Z mass spectrometer. Molecular weights were determined from benzene solutions by a vapor pressure osmometer (Mechro Lab., 301A).

The polarographic measurement were conducted on a Yanagimoto model PA-102; as a supporting electrolyte, a $0.05-N$ solution of tetraethyl ammonium iodide in a 3:1 mixture of dioxane and water was used.

\section{RESULTS AND DISCUSSION}

\section{Catalyst Screening}

Various potential catalysts were screened for 
Polymerization of Acetaldazine

Table I. Polymerization of acetaldazine by various catalysts; monomer-10.24 mmol

\begin{tabular}{|c|c|c|c|c|c|}
\hline Polymerization catalyst & Solvent & Temp, ${ }^{\circ} \mathrm{C}$ & Reacion time, hr & Yield, \% & Mol wt \\
\hline $\mathrm{BuLi}^{\mathrm{a}}$ & THF & -78 & 2 & $52.2^{\mathrm{d}}$ & 1180 \\
\hline$" 1$ & " & 0 & 2 & $72.2^{\mathrm{d}}$ & 803 \\
\hline " & Toluene & 0 & 2 & $48.4^{\mathrm{d}}$ & 884 \\
\hline $\mathrm{Na}(\mathrm{Naph})^{\mathrm{a}}$ & THF & 0 & 2 & $72.6^{\mathrm{d}}$ & 873 \\
\hline $\mathrm{BuMgBr}^{\mathrm{a}}$ & THF & 0 & 2 & $33.7^{\mathrm{d}}$ & 721 \\
\hline $\mathrm{Na}(\text { Ketyl })^{\mathrm{a}}$ & THF & 0 & 2 & $27.9^{\mathrm{d}}$ & 805 \\
\hline \multirow[t]{2}{*}{$\mathrm{LiAlH}_{4}^{\mathrm{a}}$} & THF & 0 & 20 & 0 & \\
\hline & $" \prime$ & 30 & 20 & $12.0^{\mathrm{d}}$ & 334 \\
\hline $\mathrm{MeONa}^{\mathrm{a}}$ & Hexane & 0 & 30 & 0 & \\
\hline $\mathrm{AIBN}^{\mathrm{b}}$ & THF & 60 & 48 & 0 & \\
\hline $\mathrm{BPO}^{\mathrm{b}}$ & THF & 60 & 48 & 0 & \\
\hline $\mathrm{BF}_{3} \mathrm{OEt}_{2}{ }^{\mathrm{c}}$ & $\left(\mathrm{CH}_{2} \mathrm{Cl}\right)_{2}$ & 0 & 0.5 & $7.2^{\mathrm{e}}$ & 213 \\
\hline
\end{tabular}

a $(1.2 \sim 6.0) \times 10^{-2} \mathrm{mmol}$.

b $0.61 \times 10^{-3} \mathrm{~mol}$.

c $0.86 \times 10^{-2} \mathrm{~mol} / l$.

d Water insoluble.

- Water soluble.

their ability to polymerize acetaldazine. The results are shown in Table $I$.

Acetaldazine failed to be polymerized by radical initiators. The reaction system was colored orange-red by typical anionic catalysts, and oligomers were obtained in good yields. A timeconversion curve in the polymerization catalyzed by butyllithium is shown in Figure 1 . The yield of the oligomer was $72.2 \%$ although the monomer was completely consumed after $2 \mathrm{hr}$ according to gas chromatographic analysis. Lower watersoluble oligomers may have been lost when the ether solution of the oligomer was shaken with distilled water in order to remove the inorganic

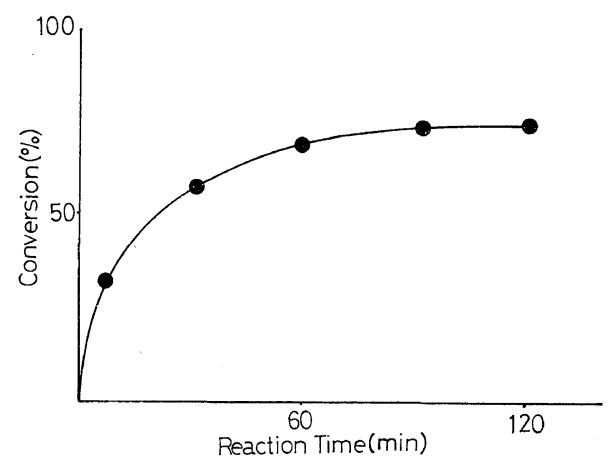

Figure 1. Time-conversion curve of water-insoluble fraction: monomer, $1.024 \mathrm{~mol} / l$; catalyst, $6.0 \times 10^{-2} \mathrm{~mol} / l$; solvent, THF; reaction temp, $0^{\circ} \mathrm{C}$. species in it.

Polymerization was also initiated by a Grignard reagent, by a metal ketyl, and gradually even by lithium aluminum hydride, in which case the color was orange-red. These results show that acetaldazine can be easily polymerized by an anionic mechanism.

When Lewis acid catalysts were also tested, oligomers were obtained in low yield. The catalyst was completely consumed after a short time. The residual acetaldazine was polymerized further when fresh catalyst was added. These results suggest that a termination reaction was very rapid, and it presumably was caused by the oligomer. $^{19}$

\section{The Structure of the Oligomer Obtained from Anionic Initiator}

The IR spectra of the oligomers obtained by various anionic catalysts were identical, with the exception of the intensities of the absorption bands. The oligomers were pale yellow to yellow viscous liquids, soluble in methanol, acetone, ether, benzene, and dimethylformamide but insoluble in aliphatic hydrocarbons. However, the low-molecular-weight oligomer below 200 was soluble in water. The number-average molecular weight of the water-insoluble oligomer was above 300. The higher-molecular-weight oligomer (above 1000) was slightly soluble in benzene and 


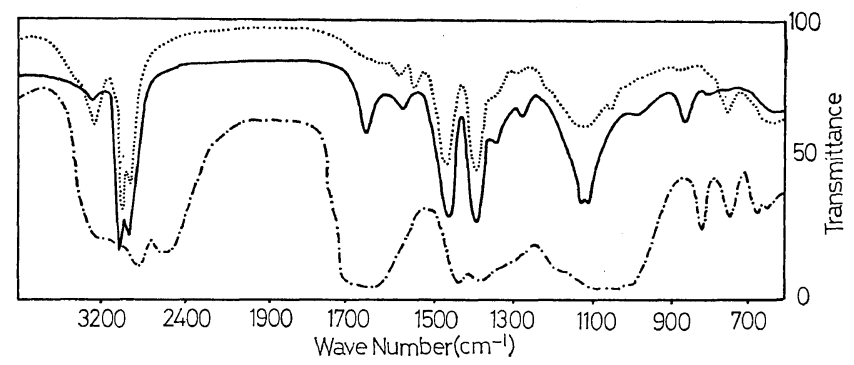

Figure 2. IR spectra of polyacetaldazine and its derivatives: (a) polyacetaldazine; (b) --.-, after reduction; (c) _..., after hydrolysis.

toluene. When the oligomer was subject to oxidation and hydrolysis, it became yellow or yellow-brown, as was shown by the increase in its oxygen content with time on contact with the air. The oligomer must be stored, therefore, with extreme care. From the elementary analysis, and by assuming that the oligomer of molecular weight 730 contained a terminal butyl group from the initiator, its structure, $\mathrm{C}_{4} \mathrm{H}_{9}\left(\mathrm{C}_{4} \mathrm{H}_{8} \mathrm{~N}_{2}\right)_{8} \mathrm{H}$ can be assigned. The IR spectrum of the oligomer is shown in Figure 2a. The absorption bands at 1645,1555 , and $865 \mathrm{~cm}^{-1}$ were assigned to $\nu(\mathrm{C}=\mathrm{N})$, $\nu(\mathrm{N}=\mathrm{N})$, and $\delta(\mathrm{N}=\mathrm{C}-\mathrm{H})$, respectively, by taking into account the change of the IR spectra before and after the chemical reactions of the oligomer. The validity of such assignments was justified by a linear correlation between the ratio of the optical densities of the $\mathrm{C}=\mathrm{N}$ and $\mathrm{N}=\mathrm{N}$ absorption bands and the ratio estimated chemically. ${ }^{20}$

The IR spectrum, after the reduction of the oligomer by aluminum amalgam, is shown in Figure 2b. The absorption bands at 1645,1555 , and $865 \mathrm{~cm}^{-1}$ decreased remarkably and the new absorption bands characteristic of the $\mathrm{NH}$ group appeared at $3200,1680,1520$, and $760 \mathrm{~cm}^{-1}$. The IR spectrum after the hydrolysis by dilute aqueous hydrochloric acid is shown in Figure 2c. The new broad absorption bands characteristic of $\mathrm{N}-\mathrm{H}$ appeared, and the modified oligomer became soluble in water. These results indicate

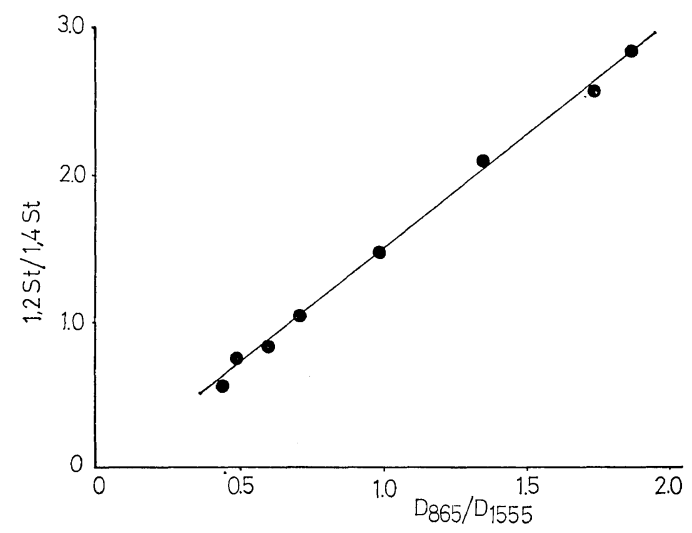

Figure 3. The correlation between the ratio of the 1,2-structure to the 1,4-structure and $D_{865} / D_{1555}$.

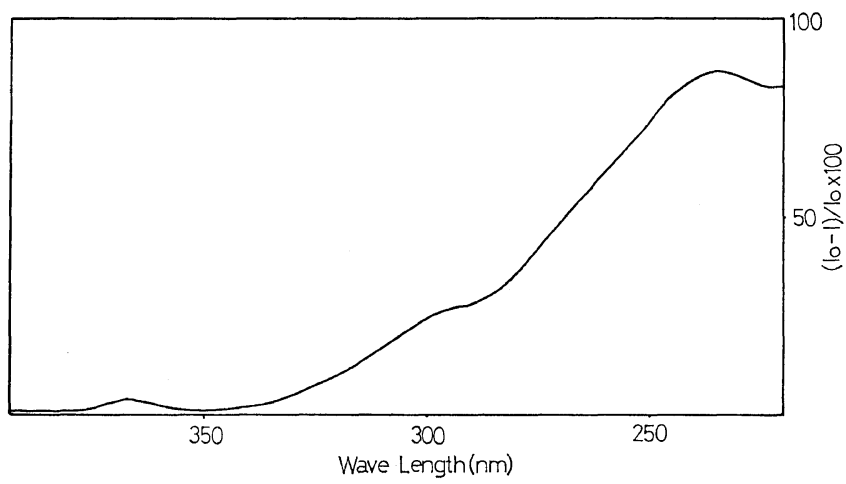

Figure 4. UV spectrum of polyacetaldazine: solvent, ethanol; polymer concentration, $0.59 \mathrm{~g} / l$. 
that the oligomer may have both the 1,2- and the 1,4-structures.

The electronic spectrum of the oligomer was measured in the range from 200 to $700 \mathrm{~nm}$. The absorption band at $238 \mathrm{~nm}$ is assigned to the $n-\pi^{*}$ transition in the $-\mathrm{C}=\mathrm{N}$ group and the band at $375 \mathrm{~nm}$ is characteristic of the $n-\pi^{*}$ transition in the $-\mathrm{N}=\mathrm{N}$ group. ${ }^{21,22}$ A shoulder at $291 \mathrm{~nm}$ is similar to the band at $285 \mathrm{~nm}$ in acetaldazine. In the course of anionic polymerization a proton transfer may occur from the acetaldazine monomer to the anion on the growing chain. The assumption that the anion obtained by this transfer can polymerize the monomer was confirmed by a larger number of oligomers than butyllithium catalyst, as shown in next section. (Table III). Therefore the shoulder at $291 \mathrm{~nm}$ is reasonably assigned to the $n-\pi^{*}$ transition at the terminal $\mathrm{CH}_{3} \mathrm{CH}=\mathrm{CH}=\mathrm{N}-\mathrm{N}=\mathrm{CH}-\mathrm{CH}_{2}$ group obtained from proton transfer.

The structure of the oligomer was further established by qualitative chemical tests for functional groups. The introduction of the volatile product obtained from the treatment of the oligomer with dilute aqueous hydrochloric acid solution into an alcoholic solution of 2,4dinitrophenylhydrazine gave acetaldehyde hydrazone, indicating the existence of the pendant $-\mathrm{N}=\mathrm{CH}-\mathrm{CH}_{3}$ groups. On hydrolysis the residual solution also gave a positive Tollen's test.

When the oligomer was stored for $3 \mathrm{hr}$ at $60^{\circ} \mathrm{C}$ under nitrogen, the optical density at $3190 \mathrm{~cm}^{-1}$ increased and that at $1555 \mathrm{~cm}^{-1}$ concomitantly decreased. This result suggests that some of the $\begin{array}{ll}\mathrm{CH}_{3} & \stackrel{\mathrm{CH}_{3}}{\mathrm{C}} \mathrm{H}-\mathrm{N}=\mathrm{N}-\stackrel{\mathrm{C}}{\mathrm{C}} \mathrm{H}-\text { units have rearranged to } \\ - & \end{array}$ the more stable $-\stackrel{{ }^{C}}{\mathrm{CH}_{3}} \mathrm{H}-\mathrm{NH}-\mathrm{N}=\stackrel{1}{\mathrm{C}}-$ units. $^{23,24}$ This rearrangement was very slow below room temperature and the change of the IR spectrum was not appreciable in $1 \mathrm{hr}$, but was apparent after 1 week.

The predominant gaseous product obtained from the pyrolysis of the oligomer at $150^{\circ} \mathrm{C}$ under high vacuum was determined by high resolution mass spectrometry to be the nitrogen molecule $(m / e=$ 28.02). This also supports the presence of the $\begin{array}{ll}\mathrm{CH}_{3} & \mathrm{CH}_{3} \\ -\stackrel{+}{\mathrm{C}} \mathrm{H}-\mathrm{N}=\mathrm{N}-\stackrel{!}{\mathrm{C}} \mathrm{H}-\text { units. }\end{array}$
From these results, the following structure of the oligomer is proposed.

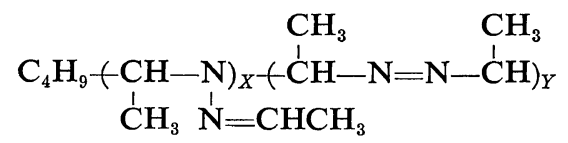

\section{Anionic Polymerizability of Acetaldazine}

Although acetaldazine is an azomethine analogue of hexadiene which is difficult to polymerize anionically, it was polymerized to the oligomer in good yields by various anionic catalysts. The rate of the anionic polymerization of acetaldazine is remarkably larger than that of hexadiene; ${ }^{25}$ thus, the anionic polymerizability of acetaldazine was qualitatively compared with that of various vinyl and diene compounds.

The polymerization of acetaldazine was realized by a Grignard reagent and a metal ketyl which did not initiate the polymerization of styrene and butadiene. $^{26}$ Acetaldazine was gradually polymerized to an oligomer even by lithium aluminum hydride, a catalyst which can polymerize methyl methacrylate. However, the polymerization of acetaldazine was not initiated by sodium methoxide, which caused the oligomerization of acrylonitrile and methyl methacrylate.

These results suggest that acetaldazine has a more anionic polymerizability than styrene and butadiene but a less anionic polymerizability than acrylonitrile and methyl methacrylate. Fueno, et al. ${ }^{27}$ suggested that the half-wave potentials of various vinyl compounds were correlated with the order of the ionic polymerizability. Therefore, the half-wave potential of acetaldazine was determined under the same conditions as Fueno, et al. These results are shown in Table II along with Fueno's data. ${ }^{27}$ The order of the anionic polymerizability predicted by the comparison of the half-wave potential of acetaldazine with those

Table II. Half-wave potential

\begin{tabular}{cc}
\hline Substance & $-E_{1 / 2}$ \\
\hline Acetaldazine & 2.04 \\
AN $^{27}$ & 2.01 \\
$\mathrm{MMA}^{27}$ & 2.01 \\
$\mathrm{St}^{27}$ & 2.35 \\
Isop $^{27}$ & 2.70
\end{tabular}

Solvent, water-dioxane (1:3).

Supporting electrolyte, tetraethyl ammonium iodide. 


\section{KAMACHI and S. MURAhashi}

Table III. The comparison of oligomer molecular weight with catalyst concentration

\begin{tabular}{cccc}
\hline $\begin{array}{c}\text { Catalyst } \\
\text { concn, } \\
\text { mmol }\end{array}$ & $\begin{array}{c}\text { Yield, } \% \\
\text { (water } \\
\text { insoluble) }\end{array} M_{n}(D P)$ & $\begin{array}{c}\text { Concn of the } \\
\text { obtained polymer, } \\
\text { mmol }\end{array}$ \\
\hline $24 \times 10^{-2}$ & 53.3 & $947(11)$ & $50 \times 10^{-2}$ \\
$36 \times 10^{-2}$ & 52.5 & $903(11)$ & $49 \times 10^{-2}$ \\
$60 \times 10^{-2}$ & 69.8 & $843(10)$ & $70 \times 10^{-2}$ \\
$120 \times 10^{-2}$ & 80.8 & $730(9)$ & $108 \times 10^{-2}$ \\
$230 \times 10^{-2}$ & 86.4 & $386(4)$ & $221 \times 10^{-2}$ \\
\hline
\end{tabular}

Monomer, $10.24 \mathrm{mmol}$; solvent, THF.

Reaction temp, $-78^{\circ} \mathrm{C}$.

Reaction time, $60 \mathrm{~min}$.

of various vinyl compounds and dienes was consistent with that observed from polymerization catalysts. This shows that the half-wave potential can be also used as a qualitative measure of the polymerizability of azine compounds.

In spite of such a high anionic polymerizability for acetaldazine, a high-molecular-weight polymer was not obtained. The proton transfer of an $\alpha$-hydrogen of the monomer is probably an important factor in limiting the increase in the molecular weight, as shown in Table III.

In conclusion, acetaldazine was polymerized to oligomers by various ionic catalysts. The polymerization was the addition polymerization through the carbon-nitrogen double bond and the obtained oligomer has 1,2- and 1,4-units. The anionic polymerizability is high and is comparable to that of methyl methacrylate. The proton transfer of the monomer is an important factor in limiting the molecular weight.

Acknowledgement. The authors are grateful to Prof. J. K. Stille and Prof. S. Nozakura for several comments.

\section{REFERENCES}

1. V. E. Shashoua, J. Amer. Chem. Soc., 81, 3156 (1959).

2. V. E. Shashoua, W. Sweeny, and R. F. Tietz, ibid., 82, 866 (1960).

3. C. King, ibid., 86, 437 (1964).

4. G. Natta, J. D. Pietro, and M. Cambini, Makromol. Chem. 56, 200 (1962).
5. T. Kashiwagi, M. Hidai, Y. Uchida, and A. Misono, J. Polym. Sci., Part B, 8, 173 (1970).

6. G. C. Robinson, ibid., Part A-1, 2, 3901 (1964).

7. N. P. Neureiter, J. Amer. Chem. Soc., 81, 2910 (1959).

8. O. Westphal and W. Eucken, Ber., 76, 1137 (1943).

9. C. G. Overberger and A. V. Digiulio, J. Amer. Chem. Soc., 80, 6562 (1958).

10. T. Curtius and E. Zinkeisen, J. prakt. Chem., 58, 310 (1898).

11. A. Weissberger, E. S. Proskauer, J. A. Riddick, and E. E. Toops, Jr., "Organic Solvents," 2nd Ed., Interscience Publishers, Inc., New York, N.Y., 1955.

12. K. Ziegler and A. Colonius, Ann., 479, 135 (1930).

13. H. Gilman and A. H. Haubein, J. Amer. Chem. Soc., 66, 1515 (1944).

14. H. Gilman, ibid., 51, 1576 (1929).

15. A. Zilkha, P. Neta, and M. Frankel, J. Chem. Soc., 3357 (1960).

16. A. V. Tobolsky, A. Rembaum, and A. Eisenberg, J. Polym. Sci., 45, 347 (1960).

17. S. Siggia, "Quantitative Organic Analysis via Functional Groups," Third Ed., John Wiley \& Sons, Inc., New York, N. Y., 1967, p 522.

18. S. Siggia, ibid., p 93.

19. The details of these results will be published separately.

20. The details will be published in another paper (Part 2 of this series).

21. H. H. Jaffe and M. Orchin, "Theory and Application of Ultraviolet Spectroscopy," John Wiley \& Sons, Inc., New York, N. Y., 1964, p 186.

22. P. C. Huang and E. M. Kosower, J. Amer. Chem. Soc., 90, 2367 (1968).

23. H. L. Lochte, W. A. Noyes, and J. R. Bailey, ibid., 44, 2556 (1922).

24. A. J. Bellamy and R. D. Guthrie, J. Chem. Soc., 3528 (1965).

25. The details of these results will be published separately.

26. T. Tsuruta and K. F. O'Driscoll, "Structure and Mechanism in Vinyl Polymerization," Marcel Dekker, New York, N. Y., 1969, p 31.

27. T. Fueno, T. Tsuruta, and J. Furukawa, Nippon Kagaku Zasshi (J. Chem. Soc. Pure Chem. Sect.), 78, 178 (1957). 